\title{
Quantized Non-Bayesian Quickest Change Detection with Energy Harvesting
}

\author{
Sinchan Biswas*, Steffi Knorn*, Subhrakanti Dey ${ }^{\dagger}$, Anders Ahlen* \\ ${ }^{*}$ Signals and Systems Division, Uppsala University, Sweden \\ ${ }^{\dagger}$ ITR, University of South Australia, Australia \\ sinchan.biswas@angstrom.uu.se,steffi.knorn@angstrom.uu.se, \\ subhra.dey@unisa.edu.au, anders.ahlen@angstrom.uu.se
}

\begin{abstract}
This paper focuses on the analysis of an optimal sensing and quantization strategy in a multi-sensor network where each individual sensor sends its quantized log-likelihood information to the fusion center (FC) for non-Bayesian quickest change detection. It is assumed that the sensors are equipped with a battery/energy storage device of finite capacity, capable of harvesting energy from the environment. The FC is assumed to have access to either non-causal or causal channel state information (CSI) and energy state information (ESI) from all the sensors while performing the quickest change detection. The primary observations are assumed to be generated from a sequence of random variables whose probability distribution function changes at an unknown time point. The objective of the detection problem is to minimize the average detection delay of the change point with respect to a lower bound on the rate of false alarm. In this framework, the optimal sensing decision and number of quantization bits for information transmission can be determined with the constraint of limited available energy due to finite battery capacity. This optimization is formulated as a stochastic control problem and is solved using dynamic programming algorithms for both non-causal and causal CSI and ESI scenario. A set of non-linear equations is also derived to determine the optimal quantization thresholds for the sensor log-likelihood ratios, by maximizing an appropriate KullbackLeibler (KL) divergence measure between the distributions before and after the change. A uniform threshold quantization strategy is also proposed as a simple sub-optimal policy. The simulation results indicate that the optimal quantization is preferable when the number of quantization bits is low as its performance is significantly better compared to its uniform counterpart in terms of average detection delay. For the case of a large number of quantization bits, the performance benefits of using the optimal quantization as compared to its uniform counterpart diminish, as expected.
\end{abstract}

\section{INTRODUCTION}

$I^{N}$ recent years, wireless sensor systems have attracted significant attention from the research community. The use of wireless sensor network (WSN) has become prevalent in the areas such as industrial process monitoring [1], smart grid [2] and environmental monitoring [3]. Due to the fact that often these sensors are located in remote locations, it becomes a significant problem to power such networks reliably with batteries. Re-installing and replenishing batteries in such a scenario is very costly. Here, renewable power sources such as solar, wind or temperature gradients can be very useful to mitigate these problems. However as the process of energy harvesting is often unpredictable and the rechargeable batteries have finite capacities, forming optimal transmission policies in sensor networks with energy harvesting capabilities constitute a non-trivial task.

Recently, there has been significant research in the domain of energy harvesting in WSN. For example, optimal energy management policies [4], power allocation policies [5], and energy efficient transmission policies [6] are analyzed for energy harvesting WSN. One of the major responsibilities of such networks is detecting the change in distributions of the underlying observations, thus detecting anomalies or predicting failures etc. There are different ways to detect these changes as discussed in the literature [7]. Quickest change detection is one form of sequential detection techniques which has been studied extensively [8]. This detection policy is used for diverse purposes like seismic event detection, health monitoring or detecting vacant radio spectrum etc. It can be applied to the observation at the individual sensors or after collecting all the information from the sensors at the FC [9]. In this paper, we focus on the latter approach.

The classical quickest change detection problem focuses on detecting a sudden change in the probability density function of a stochastic process with minimum delay, where the delay is defined as the amount of time between the actual occurrence of change in distribution and the detection of such an event. The Non-Bayesian formulation is one of the most important ones which has been studied e.g., by G. Lorden [10] and M. Pollack [11]. Let $\mathbf{X}_{k}$ denote the vector of sensor observations at time instant $k$. The standard formulation consists of a sequence of observations $\left\{\mathbf{X}_{k}, k=1,2, \ldots\right\}$ with an unknown but deterministic change point, $\lambda$. Before the change point the observation sequence $\mathbf{X}_{1}, \mathbf{X}_{2}, \ldots, \mathbf{X}_{\lambda-1}$ are assumed to be independent and identically distributed (i.i.d) and generated from the distribution $f_{0}$ whereas after the change point $\lambda$, the sequence of observations, $\mathbf{X}_{\lambda}, \mathbf{X}_{\lambda+1}, \ldots$ is drawn from distribution $f_{1}$. The goal is to detect the change point $\lambda$ to minimize the average detection delay with respect to a false alarm constraint.

In the standard frameworks of quickest change detection, the sensors can observe the signal of interest at each time slot. But, that is not the case, when the sensors are powered by rechargeable batteries due to the stochastic nature of energy availability. Research in this domain of quickest change detection with energy constraint has been reported for the Non-Bayesian [12] and Bayesian [13] frameworks in centralized settings. Similar problems have been studied in [14], [15] without energy constraints. To the best of our knowledge there has not been any research in the domain of NonBayesian quickest change detection with energy harvesting in decentralized settings. It is an interesting research problem, due to the stochastic nature of the energy harvesting process. Thus, at every time slot, each sensor has to decide whether it has enough energy in the battery for sensing and transmitting the quantized observations to the FC. It should be noted that it is crucial to find a proper quantization strategy in such a scenario, because the performance of the detection problem is sensitive to the accuracy of the information collated at the FC. We assume that the bandwidth-constrained channels 
between the sensor transmitters and the FC receiver are subject to Rayleigh fading. The channel realization influences the amount of energy required to send each quantized bit to the FC. Thus, each sensor can decide to take an observation if it has enough energy in its battery and quantize it with an appropriate number of bits depending on the corresponding channel gain. An optimal solution of such a problem is achieved by considering maximization of a finite horizon sum of an appropriate KL measure with respect to the sensing decision and the number of quantization bits for information transmission, using Dynamic Programming (DP) techniques.

The key contributions of this paper are:

- We formulate the problem of minimizing the average detection delay for Non-Bayesian decentralized quickest change detection in a fading multi-sensor system over a finite time horizon where each sensor is capable of harvesting energy from the surroundings. The problem is addressed for both Non-Causal and Causal channel state information (CSI) and energy state information (ESI).

- The optimal sensing and number of quantization bits are determined using DP.

- An analytic solution for determining the optimal quantization thresholds, when the number of quantization bits are obtained from DP, is presented.

- Finally, performance comparison in terms of average detection delay between the non-causal and the causal CSI scenario is conducted for both optimal and a heuristic uniform quantization policy. The results indicate, that the uniform strategy is useful, when the number of quantization bits is sufficiently large, but inferior to its optimal counterpart when the number of quantization bits is small.

The rest of the paper is organized as follows. In Section II, we describe the system model and formulate the optimization problem. We present the analytic formulation for optimal threshold quantization in Section III. We also propose the idea of uniform quantization as a heuristic strategy in Section III. In Section IV, we focus on finding the optimal sensing and quantization strategy by using dynamic programming for both Non-Causal and Causal CSI and ESI. Simulation results are presented in Section V. Finally conclusions are drawn in Section VI.

\section{System Model AND PROBlem Formulation}

We study a system setup as shown in Fig.1. It consists of $N$ wireless sensors, all of which measure the same physical variable and communicate with a centralized decision maker FC. If they have sufficient energy in their battery, the individual sensors decide to sense the observation signal. We assume that due to limited bandwidth availability in the channels between the sensors and FC, each sensor quantizes its observation signal and sends it to the FC. In each time slot, the FC receives the quantized observations from the sensors who have decided to transmit the information. After collating those information, the FC performs the cumulative sum (CUSUM) test based on the received information to detect a change in distribution in the observation signal similar to the CUSUM algorithm discussed in [9].

We assume that time is slotted. During each time slot the fading channel gains between the sensors and the FC are assumed to remain fixed, but change from one slot to the next in a statistically independent and identically

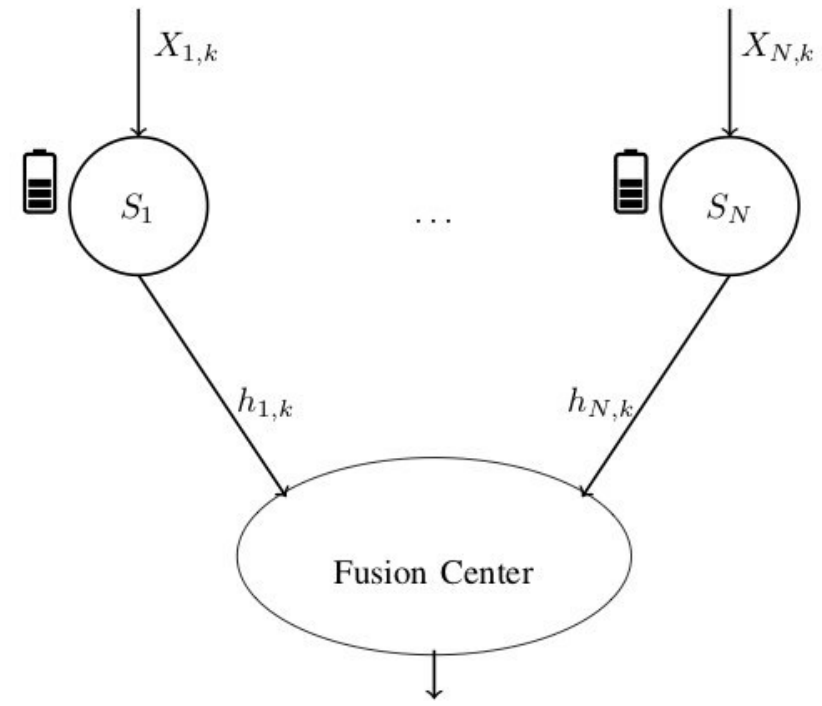

Change Point Detection

Figure 1: Quickest change detection with distributed sensors

(i.i.d) distributed manner. In the $k^{\text {th }}$ time slot the $i^{\text {th }}$ sensor decides to sense or not based on the binary parameter $\nu_{i, k}$ $1 \leq i \leq N, 1 \leq k \leq M$, where $M$ is the length of the finite time horizon under consideration for the sequential change detection problem. $\nu_{i, k} \in\{0,1\}$, where 1 (or 0 ) represents the decision to sense the observation signal (or not), respectively. Each sensor decides to sense if the amount of energy in its battery is greater or equal to the amount of energy required for sensing the observation signal and sending quantized information bits to the FC. Thus, we can write:

$$
\nu_{i, k}= \begin{cases}1, & \text { if } B_{i, k} \geq E_{s}+q_{i, k} E_{i, k}^{b} \\ 0, & \text { otherwise }\end{cases}
$$

where $E_{s}$ is the amount of energy required for sensing during each time slot, $B_{i, k}$ is the battery state, $E_{i, k}^{b}$ denotes the amount of energy required to transmit each quantized bit to the FC and $q_{i, k}$ denotes the number of such quantized bits of the $i^{t h}$ sensor, which is determined via a DP algorithm at the FC and communicated to the sensor, to be described later in Sections III and IV. We assume the finite battery capacity to be $B_{\max }$ for all sensor nodes. Then the battery state dynamics can be represented as follows:

$$
B_{i, k+1}=\min \left\{B_{\max }, B_{i, k}+H_{i, k}-E_{i, k}\right\}
$$

where $B_{i, k}$ is the amount of energy in the battery of the $i^{\text {th }}$ sensor at the $k^{\text {th }}$ time slot, $H_{i, k}$ is the amount of energy harvested and $E_{i, k}$ is the amount of energy used by the same sensor during the same time slot. This energy can be further subdivided into energy required for sensing and transmitting quantized observations as follows

$$
E_{i, k}=\nu_{i, k}\left(E_{s}+q_{i, k} E_{i, k}^{b}\right)
$$

We denote the channel gain between the $i^{\text {th }}$ sensor $S_{i}$ and the FC at the $k^{t h}$ time slot by $h_{i, k}$. Assuming either binary phase shift keying (BPSK) or quadrature phase shift keying (QPSK) modulation technique with a fixed noise power spectral density $N_{0}$, and the probability of error $P_{e}$, the energy required to transmit one quantized bit can be expressed as follows:

$$
E_{i, k}^{b}=\frac{N_{0}}{h_{i, k}}\left\{e r f c^{-1}\left(2 P_{e}\right)\right\}^{2}
$$


where $\operatorname{erfc} c^{-1}$ is the inverse complementary error function.

It should be noted that in the system model under consideration, the individual sensors don't calculate the channel gain $h_{i, k}$ and the transmission energy for each quantized bits $E_{i, k}^{b}$. They are computed at the FC, based on the pilot symbols sent to all the sensors by the FC and assuming the channel reciprocity between the sensors and FC. We denote the battery state and channel state information at $k^{t h}$ time instant by $\mathbf{B}_{k}=\left\{B_{1, k}, B_{2, k}, \ldots, B_{n, k}\right\}$ and $\mathbf{h}_{k}=\left\{h_{1, k}, h_{2, k}, \ldots, h_{N, k}\right\}$ respectively.

\section{A. Quickest Change Detection Model}

If the $i^{t h}$ sensor decides to sense during the $k^{t h}$ time slot, then the FC receives its successful transmission $X_{i, k}$. For the purpose of our discussion we assume that the observation signal is drawn from two different Gaussian distributions with the same variance but with different means, depending on whether it is received before or after the change point $\lambda$. We also assume that the change point is an unknown but deterministic time instant. We may then formulate the following hypothesis:

$$
\begin{aligned}
& \mathcal{H}_{0}: X_{i, k} \sim \mathcal{N}\left(0, \sigma^{2}\right), \quad \text { if } k<\lambda \\
& \mathcal{H}_{1}: X_{i, k} \sim \mathcal{N}\left(\mu, \sigma^{2}\right), \quad \text { if } k \geq \lambda
\end{aligned}
$$

where $\mu$ is the mean of the observation signal $X_{i, k}$ under hypothesis $\mathcal{H}_{1}$ and $\sigma^{2}$ is the variance of $X_{i, k}$ independent of any hypothesis.

Each sensor calculates the log-likelihood ratio (LLR) at each time instant which can be expressed as follows:

$$
Z_{i, k}=\frac{\mu X_{i, k}}{\sigma^{2}}-\frac{\mu^{2}}{2 \sigma^{2}}
$$

At the $k^{t h}$ time slot the sensor $S_{i}$ quantizes the LLR, $Z_{i, k}$ and corresponding $q_{i, k}$ bits encoded message $U_{i}(k)$ is sent to the FC. Let $g_{i}^{j}$ denote the probability mass function (pmf) of $U_{i}(k)$ when the observation is drawn from hypothesis $\mathcal{H}_{j}$. If we denote the $l^{\text {th }}$ quantization threshold for $i^{\text {th }}$ sensor as $t_{l}^{i}$, then these pmf's can be expressed as follows:

$$
\begin{gathered}
g_{i}^{1}(l)=\Phi\left\{\frac{t_{l+1}^{i}-\mu}{\sigma}\right\}-\Phi\left\{\frac{t_{l}^{i}-\mu}{\sigma}\right\} \\
g_{i}^{0}(l)=\Phi\left\{\frac{t_{l+1}^{i}}{\sigma}\right\}-\Phi\left\{\frac{t_{l}^{i}}{\sigma}\right\}
\end{gathered}
$$

where $\Phi$ is the cumulative distribution function of the standard normal variable.

After receiving the quantized information from the sensors, the FC computes the discrete LLR between hypotheses $\mathcal{H}_{1}$ and $\mathcal{H}_{0}$. The LLR between quantized distributions for the $k^{t h}$ time slot at the FC is denoted by $Z_{k}^{q}$. It can be written as follows:

$$
Z_{k}^{q}=\sum_{i=1}^{N} \log \frac{g_{i}^{1}\left(U_{i}(k)\right)}{g_{i}^{0}\left(U_{i}(k)\right)} .
$$

We denote $T$ as the stopping time, i.e., the time instant when the quickest change detection algorithm declares that a change has been detected in the distribution of the observation. The sensing strategy $\boldsymbol{\nu}=$ $\left\{\nu_{i, k}, i=1, \ldots, N ; k=1, \ldots, M\right\}$, quantization functions $\mathbf{q}=\left\{q_{i, k}, i=1, \ldots, N ; k=1, \ldots, M\right\}$ along with the stopping time $T$ at the FC form the policy $\phi=(\boldsymbol{\nu}, \mathbf{q}, T)$.
In the classical Non-Bayesian quickest change detection mini-max formulation, the goal is to detect the change point as soon as possible after it occurs. In other words, we have to find the quantization and sensing policy $\phi$ that minimizes the average worst case detection delay (SADD) [11], which is defined as follows:

$$
S A D D(\phi)=\sup _{1 \leq \lambda<\infty} \mathbb{E}_{\lambda}(T-\lambda \mid T \geq \lambda) .
$$

In the above expression $\mathbb{E}_{\lambda}$ denotes the expectation when the change happens at time instant $\lambda$. Once we determine the optimal sensing strategy $\nu^{\star}$ and quantization function $\mathbf{q}^{\star}$, we define $\tilde{\phi}=\left(\boldsymbol{\nu}^{\star}, \mathbf{q}^{\star}, T\right)$. Then the optimal stopping time $T^{\star}$ can be determined by the minimax change point detection procedure [9] as follows:

$$
T^{\star}=\min _{T} S A D D(\tilde{\phi}) \quad \text { s.t. } \mathbb{E}_{\infty}[T]>\gamma ; \gamma>1
$$

where $\mathbb{E}_{\infty}[T]$ denotes the stopping time declared by the algorithm when the change never occurs, i.e., $\lambda=\infty$. This quantity is called average run length to false alarm, because this indicates the rate of false alarm. It is lower bounded by a constant false alarm rate, i.e., the minimum expected duration to false alarm $\gamma>1$. We determine the solution of the optimization problem (9) by applying the CUSUM test at the FC. The CUSUM test statistics is formulated as follows:

$$
W^{q}(k)=\max \left\{0, W^{q}(k-1)+Z^{q}(k)\right\}, \quad W^{q}(0)=0 .
$$

The stopping time can be determined by the CUSUM procedure as:

$$
T^{\star}=\min \left\{k \geq 1: W^{q}(k) \geq h\right\}, \quad h=\log \gamma .
$$

\section{B. Optimization Problem Formulation}

The problem under consideration is to find the optimal sensing and quantization policy to minimize the average delay in detecting the change point. The asymptotic performance of the optimal decentralized detection procedure for a fixed sensing and quantization policy can be written as follows [9]:

$$
S A D D(T) \sim \frac{\log \gamma}{\mathbf{I}_{t o t}^{q}} \text { as } \gamma \rightarrow \infty
$$

where $\mathbf{I}_{t o t}^{q}$ is the Kullback-Leibler (KL) information number between the hypothesis $\mathcal{H}_{1}$ and $\mathcal{H}_{0}$ at the FC. In general, assuming error-free transmission between a fixed number of active sensors and the $\mathrm{FC}$, the expression $\mathbf{I}_{t o t}^{q}$ can be determined by summing individual KL information measures over the number of active sensors communicating to the FC. It is to be noted, however, that computing $\mathbf{I}_{\text {tot }}^{q}$ at the FC in our current setting is not straightforward, as it depends on a number of factors including the number of active sensors sending information to the FC in each time slot, whether the data sent from each active sensor to the $\mathrm{FC}$ are received successfully (i.e. with probability $\left(1-P_{e}\right)$ ), and the random realizations of the harvested energy and channel gains in each time slot.

Remark. For simplicity, we first make the assumption that $P_{e}$ is sufficiently small, such that an active sensor's transmitted data is received successfully at the FC with high probability when the sensor transmits with its required transmission energy per bit (3), and therefore, we ignore the effect of erroneous transmission. This can be justified very easily in the symmetric case where all sensors have the same 
$P_{e}$ requirement at the $F C$, in which case, it is easy to show that the KL information measure at the FC will be scaled by a factor of $\left(1-P_{e}\right)$.

Next, we note that the number of active sensors $n_{k}$ in the $k$-th time slot is not fixed but a random variable which depends on the harvested energy and channel state information, for a given sensing and quantization rate (which in turn determines the energy level in a sensor's battery at the beginning of a time slot) policy. For a given $n_{k}$, all else being deterministic and time-invariant, $\mathbf{I}_{\text {tot }}^{q}$ can be expressed as:

$$
\mathbf{I}_{\text {tot }}^{q}=\sum_{i=1}^{n_{k}} I\left(g_{i}^{1}, g_{i}^{0}\right)=\sum_{i=1}^{n_{k}} \sum_{l=0}^{2^{q_{i, k}}-1} g_{i}^{1}(l) \log \frac{g_{i}^{1}(l)}{g_{i}^{0}(l)}
$$

where $I\left(g_{i}^{1}, g_{i}^{0}\right)$ denotes the KL divergence between the $i^{t h}$ sensor's probability mass functions $g_{i}^{1}$ and $g_{i}^{0} \cdot 2^{q_{i, k}}-1$ denotes the number of quantization thresholds, when the number of quantization bits is chosen to be $q_{i, k}$.

Clearly, minimizing the asymptotic average worst case detection delay is equivalent to the problem of maximizing $\mathbf{I}_{t o t}^{q}$, provided that the number of active sensors $n_{k}$ and the realizations of $\mathbf{B}_{k}, \mathbf{h}_{k}$ are deterministic and identical in each slot. We are however interested in optimizing the sensing and quantization policy over a finite time horizon, where $\mathbf{B}_{k}, \mathbf{h}_{k}$ (and hence, $n_{k}$ ) vary randomly from one time slot to another. The reason behind this problem formulation is (i) to study the effects of changing battery level and channel state information in different sensors and the quickest change detection process, and (ii) in return, allowing for a more general energy harvesting and expenditure model than the binary model studied in [12]. Furthermore, given stationary and ergodic energy harvesting and fading channel processes, $\frac{1}{M} \sum_{k=1}^{M} \mathbf{I}_{\text {tot }}^{q}$ can be interpreted as the average KL measure for such a problem, as $M \rightarrow \infty$. While the asymptotic upper bound on the average detection delay is not exactly inversely proportional to the average $\mathrm{KL}$ measure, the inverse of the average KL measure still provides a lower bound on the asymptotic average detection delay from Jensen's inequality. In addition, the solution to the problem of maximizing an average $\mathrm{KL}$ measure can provide further insights into the design of optimal quantization and sensing policies for minimizing the average detection delay in the asymptotic case when the probability of false alarm goes to zero. We thus formulate an optimization problem as follows:

$$
\begin{aligned}
& \max _{\nu_{i, k}, q_{i, k}} \sum_{k=1}^{M} \mathbb{E}\left\{\sum_{i=1}^{n_{k}}\left\{\nu_{i, k} \sum_{l=0}^{2^{q_{i, k}}-1} g_{i}^{1}(l) \log \frac{g_{i}^{1}(l)}{g_{i}^{0}(l)}\right\}\right\} \\
& \text { s.t. } \nu_{i, k} \in\{0,1\} ; \forall i, k \\
& q_{i, k} \in\left\{1, \ldots, Q_{\max }\right\} ; \forall i, k \\
& E_{i, k} \leq B_{i, k} ; \forall i, k
\end{aligned}
$$

In the optimization problem the expectation $\mathbb{E}$ is taken over the random variables $\mathbf{B}_{k}$ and $\mathbf{h}_{k}$ (and hence also $n_{k}$ ). In the constraint (12), $Q_{\max }$ denotes the upper limit on the number of quantization bits $q_{i, k}$ and (11), (12) and (13) refer to the binary choice of the sensing parameter $\nu_{i, k}$, the feasibility set of number of quantization bits $q_{i, k}$, and the energy causality constraints, respectively.

\section{Non-CAusal Optimization with Finite BAtTERy}

In this section, we analyze the optimization problem with the assumption that the CSI between the sensors and the
FC along with the information about harvested energy is available non-causally. We notice that $\nu_{i, k}$ and $q_{i, k}$ are both discrete variables, and thus the problem under consideration is a combinatorial optimization problem. Therefore, the optimization problem will be solved by DP similar to the causal CSI case, which will be discussed in the next section. Clearly, this sort of non-causal setup is hardly practical, but it provides an important benchmark for performance comparison.

Remark. Note that for a fixed sensing and quantization policy with non-causal knowledge of $\left\{\mathbf{B}_{k}, \mathbf{h}_{k}\right\}$ for all $k=1,2, \ldots, M$, the objective function in the optimization problem (11)-(13) becomes deterministic. Thus, for the noncausal case, the expectation operator can be removed.

In the $k^{t h}$ time slot, once sensor $S_{i}$ decides that $\nu_{i, k}=1$ (and the optimal $q_{i, k}$ has been computed by DP off-line), the next question is how to determine the thresholds for the quantization process of the LLR. To this end, we find the quantization function $\psi_{i, k}$ that minimizes the average detection delay. Hence, we have to find $2^{q_{i, k}}-1$ thresholds $t_{0}, t_{1}, \ldots, t_{2^{q_{i, k}-1}}$, such that:

$$
\psi_{i, k}\left(X_{i, k}\right)=b_{i, k} \text { only if } t_{b_{i, k}}<Z_{i, k}\left(X_{i, k}\right) \leq t_{b_{i, k}+1}
$$

In the next two subsections we will discuss how to find the optimal quantization thresholds and a heuristic-based uniform quantization policy, respectively.

\section{A. Optimal Threshold Quantization}

Ideally speaking, the sensing decision, the number of quantization bits and the corresponding quantization thresholds should be jointly optimized using DP. However, as DP incurs exponential computational complexity, we optimize the quantization thresholds non-adaptively. This means that the number of quantization bits is computed through DP. Furthermore, the optimal quantization thresholds corresponding to each number of quantization bits are pre-computed by maximizing the following sum of KL divergence measure over a finite time horizon:

$$
\mathcal{F}\left(\left\{t_{l}: l \in\left\{0, \ldots, 2^{q_{i, k}}-1\right\}\right\}\right)=\sum_{i=1}^{n_{k}} \sum_{l=0}^{2^{q_{i, k}}-1} g_{i}^{1}(l) \log \frac{g_{i}^{1}(l)}{g_{i}^{0}(l)}
$$

when the optimal $\nu_{i, k}$ and $q_{i, k}$ satisfy (11), (12) and (13).

Suppressing the sensor index $i$ for readability, the optimal solution for the threshold $t_{l}$ can be found by solving for $\frac{\partial \mathcal{F}}{\partial t_{l}}=0$. Only two terms in the above expression are functions of $t_{l}$ as we note from (5) and (6), i.e., (for a given sensor) only $g^{1}(l), g^{0}(l), g^{1}(l-1), g^{0}(l-1)$ depend on $t_{l}$. So the gradient expression can be simplified as:

$$
\frac{\partial \mathcal{F}}{\partial t_{l}}=\frac{\partial}{\partial t_{l}}\left\{\mathcal{F}_{1}+\mathcal{F}_{2}\right\}
$$

where these two terms are $\mathcal{F}_{1}=g^{1}(l-1) \log \frac{g^{1}(l-1)}{g^{0}(l-1)}$ and $\mathcal{F}_{2}=g^{1}(l) \log \frac{g^{1}(l)}{g^{0}(l)}$. The individual gradients of these terms can be simplified as follows:

$$
\begin{gathered}
\frac{\partial \mathcal{F}_{1}}{\partial t_{l}}=\frac{\partial g^{1}(l-1)}{\partial t_{l}} \log \frac{g^{1}(l-1)}{g^{0}(l-1)}+g^{0}(l-1) \frac{\partial}{\partial t_{l}}\left\{\frac{g^{1}(l-1)}{g^{0}(l-1)}\right\} \\
\frac{\partial \mathcal{F}_{2}}{\partial t_{l}}=\frac{\partial g^{1}(l)}{\partial t_{l}} \log \frac{g^{1}(l)}{g^{0}(l)}+g^{0}(l) \frac{\partial}{\partial t_{l}}\left\{\frac{g^{1}(l)}{g^{0}(l)}\right\} .
\end{gathered}
$$


These two gradients are further simplified by replacing the individual gradients of the four cumulative distribution functions as follows:

$$
\begin{gathered}
\frac{\partial g^{1}(l-1)}{\partial t_{l}}=-\frac{\partial g^{1}(l)}{\partial t_{l}}=\frac{1}{\sigma \sqrt{2 \pi}} e^{-\frac{1}{2}\left\{\frac{t_{l}-\mu}{\sigma}\right\}^{2}} \\
\frac{\partial g^{0}(l-1)}{\partial t_{l}}=-\frac{\partial g^{0}(l)}{\partial t_{l}}=\frac{1}{\sigma \sqrt{2 \pi}} e^{-\frac{1}{2}\left\{\frac{t_{l}}{\sigma}\right\}^{2}} .
\end{gathered}
$$

Following further algebraic manipulations, the equations $\frac{\partial \mathcal{F}}{\partial t_{l}}=0$ reduce to

$$
\begin{gathered}
\frac{\partial g^{1}(l-1)}{\partial t_{l}}\left\{\log \left\{\frac{\frac{g^{1}(l-1)}{g^{0}(l-1)}}{\frac{g^{1}(l)}{g^{0}(l)}}\right\}\right\}= \\
\frac{\partial g^{0}(l-1)}{\partial t_{l}}\left\{\frac{g^{1}(l-1)}{g^{0}(l-1)}-\frac{g^{1}(l)}{g^{0}(l)}\right\} .
\end{gathered}
$$

Inserting (16) and (17) into (18) we obtain the following set of simultaneous non-linear equations:

$$
e^{-\frac{1}{2 \sigma^{2}}\left(\mu^{2}-2 \mu t_{l}\right)}=\left\{\frac{\frac{g^{1}(l-1)}{g^{0}(l-1)}-\frac{g^{1}(l)}{g^{0}(l)}}{\log \left\{\frac{\frac{g^{1}(l-1)}{g^{0}(l-1)}}{\frac{g^{1}(l)}{g^{0}(l)}}\right\}}\right\}, l=1,2, \ldots, 2^{q_{i, k}} .
$$

We can determine the optimal thresholds by solving the above mentioned equations for a fixed $q_{i, k}$ (determined using DP) by using a suitable nonlinear equation solver.

\section{B. Uniform Threshold Quantization}

In this heuristic policy, we first decide an interval of significance in the distribution of $X_{i, k}$. We assume that the probability within this interval is high enough to ignore any value of $X_{i, k}$ outside this domain, such as mean plus or minus $3 \sigma$. Furthermore, we denote the lower and upper bound of that interval by $l_{b}$ and $u_{b}$, respectively. If the number of optimal quantization bits is $q_{i, k}$, then the numbers of quantization bins and thresholds are $2^{q_{i, k}}$ and $2^{q_{i, k}}-1$, respectively. So the length of each quantization bin is: $\Delta=\frac{u_{b}-l_{b}}{2^{q_{i}, k}}$, and the $r^{t h}$ uniform threshold will be placed at $t_{r}=l_{b}+\Delta r$. It should be noted that the choice of upper and lower bound in this uniform quantization strategy influences the performance of the corresponding detection policy. Thus, it is a reasonable to assume that these two aforementioned bounds should be optimized to achieve the best performance. However, as mentioned before, we have decided to use the domain of mean plus or minus $3 \sigma$ as most of the probability mass is concentrated in that range.

It should be noted that the optimal and uniform threshold quantization can be used for the scenario involving both the non-causal and the causal CSI.

\section{Causal Optimization With Finite Battery}

In this section, we consider the more realistic case of causal CSI/ESI scenario with finite battery. The resulting problem is a finite horizon stochastic control problem and thus can be solved by applying dynamic programming algorithms.

\section{A. Information Pattern}

We assume that during each time slot, the CSI between sensor transmitters and the $\mathrm{FC}$ receiver $\mathbf{h}_{k}=$ $\left\{h_{1, k}, h_{2, k}, \ldots, h_{N, k}\right\}$ and the battery state $\mathbf{B}_{k}=$
$\left\{B_{1, k}, B_{2, k}, \ldots, B_{N, k}\right\}$ are collated by the FC using appropriate channel estimation and feedback mechanism from the sensors. Thus, the information available at the FC during the $k^{t h}$ time slot is given by the tuple $\mathbf{J}_{k}=\left\{\mathbf{h}_{k}, \mathbf{B}_{k}, \mathbf{J}_{k-1}\right\}$ and $\mathbf{J}_{1}=\left\{\mathbf{h}_{1}, \mathbf{B}_{1}\right\}$. Note that we do not consider the amount of energy consumed to carry out this feedback. However, if the amount of energy consumed due to this process is fixed in each time slot, one can easily subtract this expenditure from the available battery energy, and the presented algorithm in our paper still applies.

\section{B. Dynamic Programming Algorithm}

In this subsection, we discuss the procedure of finding the optimal sensing decision and quantization bits for energy harvesting sensors with finite battery in order to minimize the average change point detection delay when only causal CSI and ESI are available at the FC. Once again, for a fixed sensing and quantization policy, the KL information number corresponding to the $k^{\text {th }}$ time slot is given by:

$$
D\left(\boldsymbol{\nu}_{k}, \mathbf{q}_{k}\right)=\sum_{i=1}^{n_{k}}\left\{\nu_{i, k} \sum_{l=0}^{2^{q_{i, k}}-1} g_{i}^{1}(l) \log \frac{g_{i}^{1}(l)}{g_{i}^{0}(l)}\right\} .
$$

where $\boldsymbol{\nu}_{k}=\left(\nu_{1, k}, \ldots, \nu_{M, k}\right)^{T}$ is the vector of sensing parameters $\nu_{i, k}$, and $\mathbf{q}_{k}=\left(q_{1, k}, \ldots, q_{M, k}\right)^{T}$ is the vector of number of quantization bits $q_{i, k}$ respectively. Clearly, here, $n_{k}$, the number of active sensors, is a random variable varying from slot to slot depending on the harvested energy and channel gain realizations. The feasible set for the optimization variables is defined as $S=$ $\left\{\left(\boldsymbol{\nu}_{k}, \mathbf{q}_{k}\right): \boldsymbol{\nu}_{k}, \mathbf{q}_{k}\right.$ satisfy $\left.(11),(12),(13)\right\}$. In this case of a causal information pattern, the presence of the expectation operator in the objective function of the optimization problem (11) - (13) is thus strictly necessary, and the optimization problem is truly a stochastic control problem that can be solved using DP. This leads us to the main result for determining the optimal values of sensing decision $\nu_{i, k}$ and quantization bits $q_{i, k}$, described as follows:

Theorem 1. With the initial condition $\mathbf{J}_{1}=\left\{\mathbf{h}_{1}, \mathbf{B}_{1}\right\}$, the value of the finite horizon finite battery problem with causal information can be given by $V_{1}\left(\mathbf{h}_{1}, \mathbf{B}_{1}\right)$, which can be computed by the backward Bellman dynamic programming equation:

$$
\begin{array}{r}
V_{k}\left(\mathbf{h}_{\mathbf{k}}, \mathbf{B}_{\mathbf{k}}\right)=\max _{\left(\boldsymbol{\nu}_{k}, \mathbf{q}_{k}\right) \in S}\left\{D\left(\boldsymbol{\nu}_{k}, \mathbf{q}_{k}\right)+\right. \\
\left.\mathbb{E}\left\{V_{k+1}\left(\mathbf{h}_{k+1}, \mathbf{B}_{k+1} \mid \boldsymbol{\nu}_{k}, \mathbf{q}_{k}\right)\right\}\right\}
\end{array}
$$

for $1 \leq k \leq M-1$. In (21), the expectation is computed over random variables $\mathbf{h}$ and $\mathbf{B}$. The terminal condition for the algorithm is $V_{M}\left(\mathbf{h}_{\mathbf{M}}, \mathbf{B}_{\mathbf{M}}\right)=\max _{\left(\boldsymbol{\nu}_{M}, \mathbf{q}_{M}\right) \in S} D\left(\boldsymbol{\nu}_{M}, \mathbf{q}_{M}\right)$.

Proof. See [16] for a proof.

Clearly, the optimal $\left\{\boldsymbol{\nu}_{k}, \mathbf{q}_{k}\right\}$ can be computed numerically by searching over discretized values of the optimization variables as follows:

$$
\begin{array}{r}
\left\{\boldsymbol{\nu}_{k}^{\star}, \mathbf{q}_{k}^{\star}\right\}=\underset{\left(\boldsymbol{\nu}_{k}, \mathbf{q}_{k}\right) \in S}{\operatorname{argmax}}\left\{D\left(\boldsymbol{\nu}_{k}, \mathbf{q}_{k}\right)+\right. \\
\left.\mathbb{E}\left\{V_{k+1}\left(\mathbf{h}_{k+1}, \mathbf{B}_{k+1} \mid \boldsymbol{\nu}_{k}, \mathbf{q}_{k}\right)\right\}\right\}
\end{array}
$$




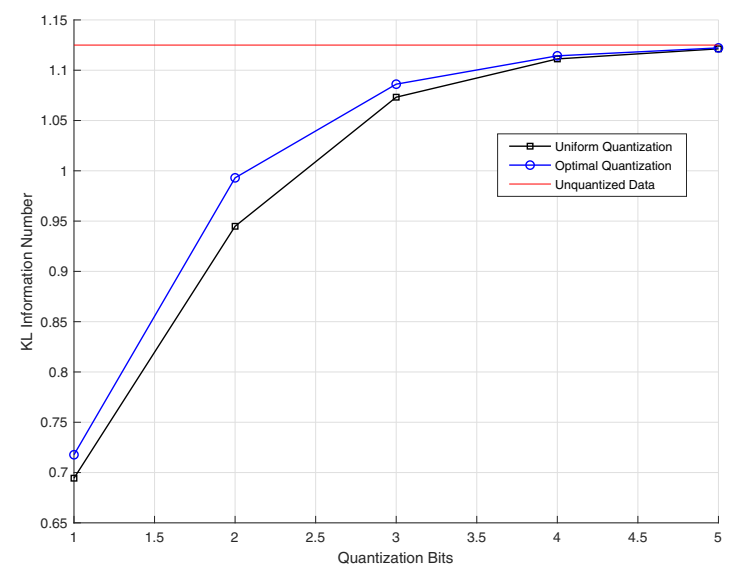

Figure 2: Single slot single user KL information number for optimal, uniform quantization and unquantized policy

This procedure is performed offline based on the statistical distribution of the channel gains and harvested energy processes. Based on this procedure, the FC creates a look-up table for optimal sensing decisions $\nu_{i, k}$ and quantization bits $q_{i, k}$ corresponding to discretized values of $\mathbf{h}_{k}$ and $\mathbf{B}_{k}$. In real time, the FC receives the causal channel gain and battery state information from the sensors and looks for the closest values of each quantity in the look-up table. The optimal values are fetched corresponding to those closest discretized parameters from the look-up table and sent to the individual sensors through feedback channels.

\section{Simulation Results}

We present simulation results for both non-causal and causal CSI scenarios with finite battery with optimal and uniformly quantized information. The channel power gain between the sensor transmitter $S_{i}$ and the FC receiver, $h_{i, k}$ is modeled as an exponentially distributed random variable with unit mean. The energy harvesting process is also assumed to be exponentially distributed with unit mean. It should be noted that the channel gain being i.i.d from one time slot to another, is assumed for its simplicity. However, the optimization problem under consideration is not constrained by more general assumption of the non i.i.d scenario. In that case, both channel gains and harvested energy can be modeled by a discrete Markov chain with a transition matrix as in [17]. We choose $\mu=1.5$ and $\sigma^{2}=1$ for the relevant distributions. The probability of bit error for transmission of quantized information is assumed to be $P_{e}=0.005$. The noise power spectral density is $N_{0}=0.02 \mu \mathrm{W} / \mathrm{Hz}$. The sensing energy is $E_{s}=0.1 \mu \mathrm{J}$. In all simulations we assume the number of sensors to be $N=2$. The maximum limit on number of quantization bits is $Q_{\max }=5$. The choice of the value of $Q_{\max }$ is motivated by Fig.2, which clearly shows that the KL information number corresponding to both optimal and uniform quantization policy and unquantized case become virtually equal when $q_{i, k}=5$. The initial battery level in all sensors is assumed to be $0.4 \mu \mathrm{J}$. For the DP implementation both battery state $B_{i, k}$ and channel power gain $h_{i, k}$ are quantized to 4 different discrete levels. The averages are taken over $10^{4}$ Monte-Carlo simulations for plots in Fig.3-Fig.6.

Fig.2 shows the comparative plot of the KL information number (14) for a single sensor case between the optimal, uniform and unquantized case with respect to the number of quantization bits. The KL information number for the

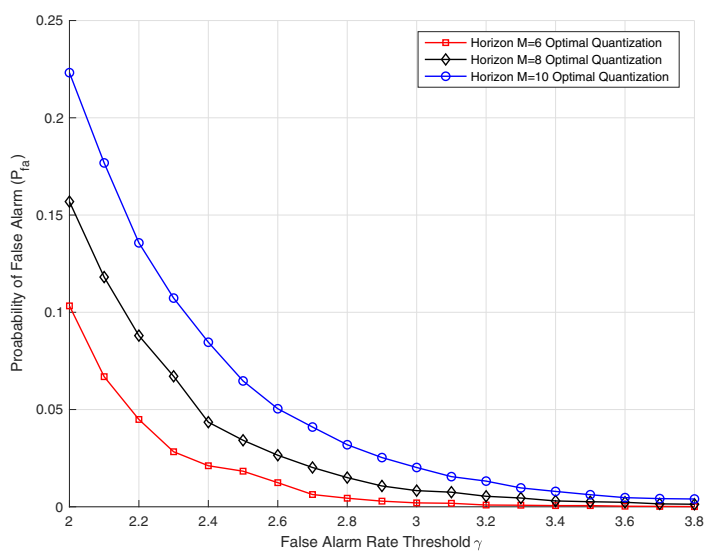

Figure 3: Probability of false alarm $P_{f a}$ vs false alarm rate bound $\gamma$ for non-causal CSI with optimal quantization policy for Horizon length $M=6,8,10$

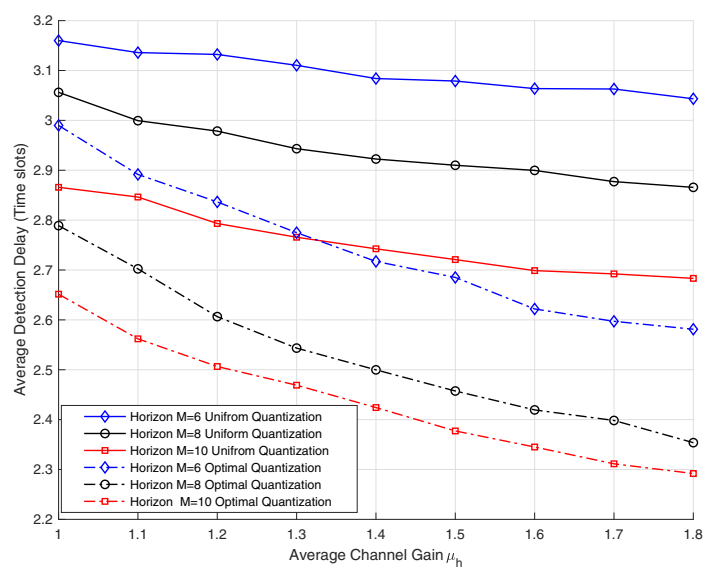

Figure 4: Average detection delay (Time slots) vs mean channel gain $\mu_{h}$ for non-causal CSI with optimal and uniform quantization policy for horizon length $M=6,8,10$

unquantized information case is found to be $\frac{\mu^{2}}{2 \sigma^{2}}$, which constitutes the upper bound for all quantized information scenarios. As expected, the optimal quantization policy performs better compared to its uniform counterpart. The KL information numbers of all three policies become comparable as the number of quantization bits increases.

Fig. 3 depicts the actual probability of false alarm, $P_{f a}$, vs the false alarm rate lower bound $\gamma$ for non-causal CSI with optimal quantization for horizon length $M=6,8,10$ respectively. We notice that the probability of false alarm increases with increasing horizon length for small $\gamma$. This is due to the fact the average detection delay decreases with increasing horizon length, which leads to decrease in expected time to false alarm. This results in an increase of probability of false alarm with increasing horizon length. For our simulations, illustrated in Fig.5-Fig.6, we fix the probability of false alarm to be $P_{f a}=0.01$. Corresponding values of $\gamma$ are determined from the plots and used in the simulations below. We use similar plots for optimal and uniform quantization for both the causal and the non-causal CSI scenario.

Fig.4 shows the average detection delay of change point $\lambda$ with respect to the mean channel gain $\mu_{h}$ for the non-causal CSI scenario, when the battery capacity $B_{\max }$ is fixed to $0.8 \mu \mathrm{J}$. We note from Fig.4 that the optimal quantization policy is more sensitive to the channel gain compared to its uniform counterpart and it has the ability to exploit 


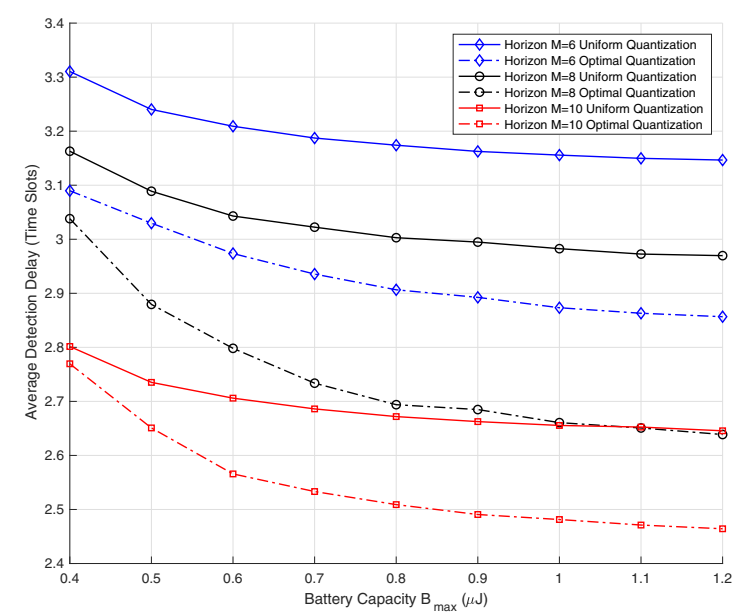

Figure 5: Average detection delay (Time slots) vs battery capacity $B_{\max }$ for non-causal CSI with optimal and uniform quantization policy for horizon length $M=6,8,10$

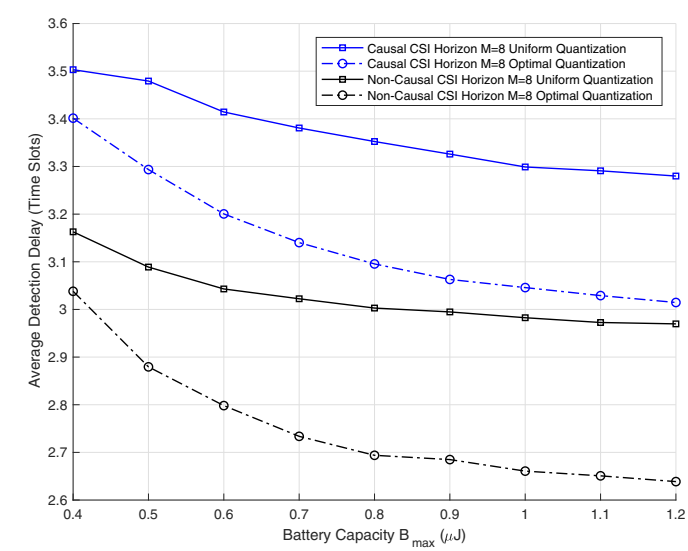

Figure 6: Average detection delay (Time slots) vs battery capacity $B_{\max }$ for non-causal and causal CSI with optimal and uniform quantization policy for horizon length $M=8$

that property when the channel gain is significantly higher. Fig.5 shows the average detection delay with respect to the battery capacity $B_{\max }$ for the non-causal CSI scenario. As a numerical comparison, we note that for $M=10$ and $B_{\max }=0.7 \mu \mathrm{J}$, the average detection delay corresponding to the non-causal CSI scenario in the uniform quantization policy is 5.9 percent higher compared to its optimal counterpart. We also notice that the average detection delay decreases with increasing horizon length, since if the information regarding CSI/ESI is non-causally available, then sensors will be able to plan its quantization and sensing strategy better as the horizon length increases.

Finally Fig.6 shows the comparative plots of the average detection delay with respect to a varying battery capacity, $B_{\max }$, for both non-causal and causal CSI scenario with the optimal and uniform quantization policies and horizon length $M=8$. As a numerical comparison, it can be seen that for $M=8$ and $B_{\max }=0.7 \mu \mathrm{J}$, the average detection delay corresponding to the optimal quantization policy in the causal CSI scenario is 15.3 percent higher compared to its non-causal counterpart. Similar plots can be obtained (but excluded due to space limitations) with respect to varying the mean channel gain $\mu_{h}$ while keeping the battery capacity $B_{\max }$ fixed.

\section{CONCLUSIONS}

This paper focuses on the problem of minimizing the detection delay of a change point in the quickest change detection framework for multi-sensor system powered by energy harvesting. The optimal sensing and quantization strategy is formulated as a stochastic control problem in both the non-causal and the causal CSI scenario and solved using dynamic programming. The non-causal CSI scenario provides a benchmark of average detection delay for the more practical causal CSI scenario. We also compute the optimal quantization thresholds once the number of quantization bits are obtained from dynamic programming, by optimizing a suitable KL divergence measure involving the quantized variables. We compare this optimal threshold policy with the uniform threshold policy and find that the optimal policy performs significantly better for lower quantization bits, whereas for higher quantization bits the performance benefits of using the optimal quantization thresholds are marginal compared to its uniform counterpart.

\section{REFERENCES}

[1] V. C. Gungor and G. P. Hancke, "Industrial wireless sensor networks: Challenges, design principles, and technical approaches," IEEE Transactions on Industrial Electronics, vol. 56, no. 10, pp. 4258-4265, Oct 2009.

[2] V. C. Gungor, B. Lu, and G. P. Hancke, "Opportunities and challenges of wireless sensor networks in smart grid," IEEE Transactions on Industrial Electronics, vol. 57, no. 10, pp. 3557-3564, Oct 2010.

[3] I. F. Akyildiz, W. Su, Y. Sankarasubramaniam, and E. Cayirci, "A survey on sensor networks," IEEE Communications Magazine, vol. 40, no. 8, pp. 102-114, Aug 2002.

[4] V. Sharma, U. Mukherji, V. Joseph, and S. Gupta, "Optimal energy management policies for energy harvesting sensor nodes," IEEE Transactions on Wireless Communications, vol. 9, no. 4, pp. 1326-1336, April 2010.

[5] Z. Ding, S. M. Perlaza, I. Esnaola, and H. V. Poor, "Power allocation strategies in energy harvesting wireless cooperative networks," IEEE Transactions on Wireless Communications, vol. 13, no. 2, pp. 846860 , February 2014.

[6] M. Gregori and M. Payaro, "Energy-efficient transmission for wireless energy harvesting nodes," IEEE Transactions on Wireless Communications, vol. 12, no. 3, pp. 1244-1254, March 2013.

[7] S. Kay, Fundamentals of Statistical Signal Processing: Detection theory, ser. Prentice Hall Signal Processing Series. Prentice-Hall PTR, 1998.

[8] H. Poor and O. Hadjiliadis, Quickest Detection. Cambridge University Press, 2008.

[9] A. G. Tartakovsky and V. V. Veeravalli, "Asymptotically optimal quickest change detection in distributed sensor systems," Sequential Analysis, vol. 27, no. 4, pp. 441-475, 2008.

[10] G. Lorden, "Procedures for reacting to a change in distribution," Ann. Math. Statist., vol. 42, no. 6, pp. 1897-1908, 121971.

[11] M. Pollak, "Optimal detection of a change in distribution," Ann. Statist., vol. 13, no. 1, pp. 206-227, 031985.

[12] J. Geng and L. Lai, "Non-bayesian quickest change detection with stochastic sample right constraints," IEEE Transactions on Signal Processing, vol. 61, no. 20, pp. 5090-5102, Oct 2013.

[13] J. Geng, E. Bayraktar, and L. Lai, "Bayesian quickest change-point detection with sampling right constraints," IEEE Transactions on Information Theory, vol. 60, no. 10, pp. 6474-6490, Oct 2014.

[14] G. V. Moustakides, "Decentralized cusum change detection," in Information Fusion, 2006 9th International Conference on. IEEE, 2006,

pp. 1-6.
[15] V. V. Veeravalli, "Decentralized quickest change detection," IEEE Transactions on Information Theory, vol. 47, no. 4, pp. 1657-1665, May 2001.

[16] D. P. Bertsekas, Dynamic Programming and Optimal Control, 2nd ed. Athena Scientific, 2000.

[17] S. Knorn and S. Dey, "Optimal energy allocation for linear control with packet loss under energy harvesting constraints," Automatica, vol. 77, pp. 259-267, 2017. 